\title{
Addressing the challenge of child undernutrition: are we ready?
}

\author{
Hiranya S Jayawickrama \\ Child Nutrition Unit, Family Health Bureau, Ministry of Health, Sri Lanka \\ *Correspondence: senanij@hotmail.com Diphttps://orcid.org/0000-0002-0462-7914 \\ DOI: https://doi.org/10.4038/jccpsl.v26i2.8260
}

Received on 13 Febuary 2020

Accepted on 07 March 2020

\section{Introduction}

Despite being a developing country, Sri Lanka has achieved remarkable success in reducing morbidity and mortality and in maintaining impressive health indices on par with developed countries. Yet, a comparable improvement in nutrition indicators of children underfive has not been apparent over the years (1-3). Stunting, wasting and underweight among children under-five remain stagnant (17.3\% vs. $17.3 \%, 14.7 \%$ vs. $15.1 \%$ and $21.1 \%$ vs. $20.5 \%$ respectively between 2006 and 2016) and are considered a 'medium' public health problem with regards to stunting and 'very high' public health problem with regards to wasting (4).

There is a global commitment to achieve Sustainable Development Goal (SDG) targets by 2030, in which target 2.2 under 'Zero Hunger' is on 'end all forms of malnutrition by 2025' to reach the internationally agreed targets on stunting and wasting in under-5 children. Targets set for Sri Lanka include stunting to be reduced to $10.8 \%$ by 2025 and $10 \%$ by 2030; and wasting to be reduced to $5 \%$ by 2025 and maintained below. However, considering the current high prevalence of stunting and wasting in the country, these targets may appear rather ambitious and unachievable.

\section{What is being done at present?}

Sri Lanka is implementing all relevant evidencebased nutrition specific/direct nutrition interventions to ensure maternal and child nutrition island wide through the well-established preventive and curative health systems. As the focal point for maternal and child health (MCH) in the Ministry of Health, the Family Health Bureau (FHB) has incorporated growth monitoring and promotion into the integrated package of $\mathrm{MCH}$, through which promotion of breastfeeding and appropriate complementary feeding, growth monitoring at specified intervals, vitamin A supplementation, home fortification of complementary food with multiple micronutrients, Zn supplementation in management of diarrhoea, management of severe and moderate acute malnutrition with therapeutic and supplementary food respectively, de-worming. etc. are carried out. These are well-supported by various other technical units of the Ministry of Health through implementation of the Food Act and iodization of salt (Environment Occupational Health and Food Safety Unit), periodic nutrition assessments (Medical Research Institute), Food Based Dietary Guidelines and Nutrition Policy (Nutrition Division) and nutrition promotion activities (Health Promotion Bureau).

The interventions implemented encompass the lifecycle across four levels of the health system operating in Sri Lanka; home/community with domiciliary care provided by public health midwives (PHMs), field MCH clinics, first contact health facilities such as central dispensaries and divisional hospitals, and referral hospitals from base hospitals upwards to teaching hospitals. The key categories of activities required to obtain a high coverage of these interventions are advocacy, capacity development and resource mobilization, logistic management, system strengthening, programme communication, and monitoring and evaluation (M\&E). These are being implemented island wide and as a result, some of the interventions have shown 
steady improvement over the years; early initiation of breastfeeding from 83.3\% (2006) to 89.7\% (2016); exclusive breastfeeding from $75.8 \%$ (2006) to $82 \%$ (2016); and minimum acceptable diet from 67.9\% (2006) to $72.3 \%$ (2016) (5). At present, the coverage of growth assessment and vitamin A supplementation is well above $80 \%$ (6). The country has a very supportive policy environment and legislative framework; National Nutrition Policy of 2010, Maternal and Child Health Policy of 2012, Food Act, Sri Lanka Code for Promotion, Protection and Support of Breastfeeding and Marketing of Designated Products, maternity protection legislations to name a few, which are supported by relevant strategy documents such as Infant and Young Child Feeding (IYCF) Strategy, technical guidelines and circulars developed to provide guidance during service provision. In addition, diverse nutrition sensitive/ indirect nutrition interventions are being implemented by the non-health sector to reduce poverty, improve food security and create a conducive environment for improved nutrition, such as poverty alleviation programmes (e.g. Samurdhi), price polices, maternity benefits to support breastfeeding and education opportunities for women. Despite all these efforts, child malnutrition indicators in the country have not improved on par with other health and social indicators.

\section{What is amiss and what more to do?}

Further reduction of malnutrition remains a huge challenge. It appears that the country has come to a point where an in-depth study into the problem is required to meet the challenges.

\section{Effective coverage of interventions}

Coverage of the interventions combined with quality ("effective coverage") is an important prerequisite to achieve a desired impact. For the same, it is required to achieve "universal coverage" of $99 \%$. It is observed that many of the child nutrition interventions are yet to achieve universal coverage predominantly due to bottle necks including resource constraints and the quality of services that is not as desired. In addition, with regard to equity, disparities in health and nutrition impact indicators, intervention coverage and service provision are still observed between estate, rural and urban sectors, among districts and specific population groups. Thus, it is imperative to increase the availability and access to child nutrition services of high quality, while creating a demand for these services and improving the knowledge of the community to bring about a positive behavioural change. To achieve these, a set of key activities needs to be carried out, such as advocacy, capacity development, resource mobilization in terms of human, financial, material resources, supervision, monitoring and evaluation. The overarching principals of governance, accountability and system strengthening are also crucial for successful conduct of these key action areas.

\section{Fine-tuning service delivery}

The key intervention in child nutrition is growth monitoring and promotion, which aim at early detection of problems and timely interventions through ageappropriate feeding counselling. Considering the strong behavioural component of undernutrition, efforts to establish appropriate feeding practices has to be initiated from the very onset when child feeding behaviours are amenable to change, rather than waiting till inappropriate feeding practices are established, leading to problems more difficult to manage. Sri Lanka is now at a phase where individual attention to prevent early faltering of growth should go hand in hand with the general interventions to promote nutrition, while ensuring that every child's growth potential is achieved to make our children well nourished, healthy and productive.

Methodologies for the delivery of this key intervention have to be re-visited with regard to their quality as well as coverage, as given below.

The ability of the system to identify and reach the target group focusing on early identification of the growth problems - While providing the baseline services for all children, the system should identify those with growth faltering very early and actively look for the underlying reasons. The system should be strengthened in such a way that our health system reaches out to every single child.

Reaching the target group on time with the most appropriate intervention - The system should be able to identify the most appropriate intervention for children with early growth faltering (when the interventions are easiest to implement and most effective) and deliver it then and there before a vicious behavioural cycle not amenable to changes begins. 
Ability to motivate the target group to bring about the desired behavioural change - The system should be able to market the concept of addressing growth faltering at the very onset as an important intervention for improving the health of community. The health staff should be capable of empowering the public in taking the initiative to adopt nutrition promoting behavioural changes.

The ability of our present healthcare system to fulfil these requirements needs to be re-visited at length. Be the health seeker attending the government sector or private sector, is an urban, rural or estate dweller, a child from the super-rich or the extreme poor, does our system reach and identify their growth potential and enhance it to the maximum is the question. Is our health staff from top level professionals to the grass root level worker knowledgeable, skilled and vigilant enough to identify the growth potential of a child and the subtle changes in growth and motivated enough to spend time, identify the cause and the correct intervention and implement it in a timely manner are questions that need introspection.

\section{Human resources for service delivery}

Inadequate human resources in the preventive health system has led to an overburdening of these service providers with many activities and programmes. Further aggravating the problem, many activities outside the scope of MCH make the health staff give less priority to nutrition related activities. Lack of financial inducements and the demanding nature of the work in terms of time, effort and scope are further barring competent individuals from entering these services, thus creating a maldistribution of human resources between curative and preventive health sectors. This situation in turn has affected the quality of services including monitoring, mentoring and supervision which are integral components of quality service provision.

There is a felt need to improve the basic training programmes, to reorient the postgraduate training to address these challenges, streamline the in-service training/ capacity building programmes and to increase the capacity of districts to roll out these programmes effectively. Imparting the ever-increasing knowledge base as depicted by the increasing number of training programmes needs to be considered along with constraints in rolling out training programmes from the national level through the district level to the $\mathrm{MOH}$ level. Ensuring quality is a context where too many programmes are being expected to be carried out by limited staff without compromising service delivery is a challenge. At the same time, the people's expectations are fast changing, as shown by an increasing number receiving services from the private sector - hospitals and individual practitioners. Ensuring that accurate and uniform messages are given to the public adhering to the national guidelines through all these institutions is a timely need.

Inadequacy of a united effort to implement the national guidelines and the recommended practices and less enthusiasm among some medical professionals to promote correct nutrition practices both in the government and private sector remains a challenge.

\section{Financial constraints}

Compared to the curative sector, the preventive health sector appears to receive less funds leading to many logistic and other constraints in service provision. Preventive care expenditures were found to be only $3 \%$ of the national current health expenditure throughout the three-year period 2014-2016 (8).

\section{Emerging threats/ challenges}

The fast changing socio-cultural environment such as lifestyle changes including more women seeking employment, urbanization and commercialization, changing family structures from extended to nuclear families with less support for child care, changing food habits which are not always supportive of healthy eating, and adverse media influence are some of the emerging challenges faced. The inability of the health sector to counteract some of these issues i.e. competing with advertising campaigns by multi-national food and beverage companies, mainly due to lack of adequate funds remains a challenge. Consequently, various myths and taboos still continue to play a role in inappropriate feeding practices.

\section{Multi-sector coordination and collaboration}

Because of its complexity, nutrition is not the responsibility of only the health sector but of many other stakeholders from non-health sector. Therefore, to bring all relevant stakeholders together, the National Nutrition Council chaired by His Excellency the President and the National Nutrition Secretariat were 
established; and 'Sri Lanka - a Nourished Nation by 2016' - a multi-sector action plan to improve nutrition was developed for strengthening the multi-sector approach. However, the desired outcome was difficult to achieve in many of the districts. Thus, inter-sectoral collaboration to improve nutrition continues to be inadequate and challenging to establish.

\section{Facing the challenges}

First and foremost, it is imperative to maintain the successes achieved so far and to increase the effective coverage of interventions to the level of 'universal coverage', while ensuring the equity of services through fewer inter-district and inter-sectoral disparities.

\section{Human resource development}

In the present system, the key officer responsible for growth monitoring at field level is the PHM guided by the supervisory staff (supervising PHM, public health nursing sisters, $\mathrm{MOH}$ ) while growth promotion through nutrition counselling is carried out during home visits, at the field weighing posts, child welfare clinics and nutrition clinics of the MOHs. In addition, regular standard health education sessions in the form of classes for caregivers on breastfeeding and complementary feeding at specified intervals are conducted in the community. Adequate and competent health care workers are essential for providing these services. To this end, it is essential to make public health more attractive; by providing incentives and adequate facilities including transport for creating an enabling working environment and thereby attract more healthcare personnel to preventive health services.

Addressing nutrition problems through nutrition counselling necessitates a fair amount of time spent on it in addition to the knowledge and skills, dedication and positive attitudes from health staff. Thus, the burden of nutrition counselling should be equally borne by doctors as well as by PHMs. As the grass root level care provider, PHMs should be able to detect and correct early problems, while the doctors should shoulder the burden of managing longstanding and complicated cases and guide PHMs and strengthen their capacities.

Ensuring regular basic training programmes, filling the existing vacancies as well as revising cadre to meet the rising demands are important for strengthening service provision. It is essential that the basic curricular of relevant disciplines (medical, nursing, midwifery etc) are revised and child nutrition including IYCF are given due prominence to ensure knowledge as well as skills of these healthcare providers. The commencement of a postgraduate course in human nutrition with a master's degree and MD a few years back by the PGIM is expected to improve the quality of services.

The changing dynamics of the health care delivery also need to be considered with many parents increasingly opting for private sector services. The quality of growth monitoring and the nutrition counselling offered in the private sector needs to be evaluated, while advocacy and capacity building on child nutrition also needs to be strengthened through regulatory mechanisms. Therefore, a collaborative effort between all the stakeholders is urgently called for in order to improve the quality of service delivery in an equitable manner.

\section{Community empowerment}

Behaviour modification and empowering the community on appropriate feeding practices is essential in creating healthy norms and feeding habits in the community. In the backdrop of fast changing sociocultural environment, avenues to bring about a positive behaviour change needs to be explored with innovative SBCC strategies. Raising public awareness and motivating them to adopt nutrition promoting behavioural changes is a must. The responsibility of mobilizing the public to take the initiative invariably falls on the health staff who should take the lead role for providing the public with correct information and guidance on feeding practices and nurturing care. Adverse media influences and commercial interests of confectionary, fast food and formula milk industries are better addressed by empowerment of the community with the support of a strong legislative framework.

\section{Strengthening the multi-sector approach}

Creating a supportive environment ensuring food security including availability and promotion of affordable healthy options for health and nutrition is the responsibility of all relevant stakeholders, though the role of the health sector in providing technical guidance should not be undermined. Apart from direct 
health interventions, a multi-pronged approach with a concerted effort by all relevant non-health stakeholders is crucial to cater to the complex socio-cultural and economic needs of the vulnerable communities such as the estate sector, populations living in poverty including the urban poor.

The importance of strengthening law enforcement and revising the existing laws and regulations namely the Food Act and the Breastfeeding Code to meet the emerging challenges should be a priority, including the addressing of disparities in maternity benefits between the government and private sector. Efforts to persuade industry to come up with healthier food options and to fall in line with national guidelines and legislations need to be strengthened.

\section{Conclusion}

An individual as well as a concerted effort is needed to address these challenges from advocacy to ground level implementation. Within the government health sector more support from health administration in strengthening the health system including the provision of adequate and timely resources from the central level to the periphery especially for the preventive health sector is required. All professional bodies, developmental partners and non-governmental organizations need to be unbiased and genuinely motivated to work towards achieving a common goal. It is imperative that all stakeholders uphold and adhere to policies, guidelines, legislations which have been developed based on hard scientific evidence. Streamlining the nutrition sensitive interventions to be implemented by the non-health sector stakeholders in order to reach the correct target groups effectively including measures to improve food security needs clearly defined roles and responsibilities, a robust coordination and monitoring mechanism at national level with technical inputs from the Ministry of Health.

\section{Author Declarations}

Acknowledgements: Dr Achini Thilakaratne (Medical Officer, Child Nutrition Unit, Family Health Bureau).

\section{References}

1. Department of Census and Statistics (DCS). Sri Lanka Demographic and Health Survey 2016. Colombo; Ministry of Health \& Indigenous Medical Services, 2017.

2. Department of Census and Statistics (DCS). Sri Lanka Demographic and Health Survey 2006-07. Colombo: Ministry of Healthcare and Nutrition, 2009.

3. Medical Research Institute \& UNICEF. National Nutrition and Micronutrient Survey 2012. Colombo: Ministry of Health, 2012.

4. de Onis M, Borghi E, Arimond M, Webb P, Croft T, Saha K, De-Regil LM, Thuita F, Heidkamp R, Krasevec J, Hayashi C, Flores-Ayala R. Prevalence thresholds for wasting, overweight and stunting in children under five years. Public Health Nutrition 2018; 22(1): 175-179.

5. Senarath U, Siriwardana I, Partheepan K. Infant and young child feeding practices in Sri Lanka: an in-depth analysis of the 2006 and 2016 Demographic and Health Surveys. Colombo: Ministry of Health (unpublished).

6. FHB. eRHMIS. Family Health Bureau, Ministry of Health, Sri Lanka. Available from: https:// www.erhmis.fhb.health.gov.lk/erhmis/dhis-webcommons/security/recovery.action

7. Ministry of Health. National Health Accounts Sri Lanka 2014, 2015. Colombo: Ministry of Nutrition and Indigenous Medicine, 2016. 